persisted as aggregates, resistant to degradation. Fibronectin aggregates within MS lesions contribute to failure of remyelination and are potential therapeutic targets for promoting remyelination. (Stoffels JMJ, de Jonge JC, Stancic M, et al. Fibronectin aggregation in multiple sclerosis lesions impairs remyelination. Brain 2013 Jan;136(Pt 1):116-31) (Response: Dr Wia Baron. E-mail: w.baron@umcg.nl).

\title{
CHILDHOOD OBESITY AND RISK OF PEDIATRIC MS
}

Researchers at Kaiser Permanente of Southern California studied a possible relation between childhood obesity and pediatric-onset multiple sclerosis (MS) or its potential precursor, clinically isolated syndrome (CIS), which encompasses optic neuritis (ON) and transverse myelitis (TM). Seventy-five newly diagnosed pediatric cases of MS or CIS were identified between 2004 and 2010; 41 (55\%) were girls, and 54 (72\%) were age 11-18. Onset of MS/CIS was uncommon at ages $2-11$ years. Thirty-eight $(50.7 \%)$ children or adolescents with MS/CIS were overweight or obese. Obesity was associated with a significantly increased risk of MS/CIS in girls but not in boys. Moderately and extremely obese patients were more likely to present with TM compared with normal/overweight children $(\mathrm{p}=0.003)$. (Langer-Gould A, Brara SM, Beaber BE, Loebnick C. Childhood obesity and risk of pediatric multiple sclerosis and clinically isolated syndrome. Neurology 2013 Feb 5;80(6):548-52). (Response: Dr Langer-Gould. E-mail: Annette.M.Langer-Gould@kp.org).

COMMENT. Childhood obesity is independently associated with an increased risk of pediatric-onset MS/CIS in girls but not in boys. The authors speculate that the rapid rise and high estrogenic exposure of obese, peripubescent girls coupled with inflammatory mediators released by adipose tissue accelerate MS/CIS onset in adolescence. Pregnancy in females and tobacco smoke among males (Palacios N, et al. Ann Epidemiol 2011 Jul;21(7):536-42), additional potential risk factors for MS, were not addressed in this study. The need to further address the progress of the childhood obesity epidemic is stressed, especially in girls.

\section{PERINATAL DISORDERS}

\section{MELATONIN AND EXPERIMENTAL PERINATAL ASPHYXIA}

Researchers from University College London, Hopital Robert Debre, and Universite Paris Diderot, Paris assessed the neuroprotective effects of melatonin combined with therapeutic hypothermia after transient hypoxia-ischemia in a piglet model of perinatal asphyxia. Melatonin administered intravenously 10 min after transient hypoxia-ischemia and repeated at $24 \mathrm{hr}$ augments hypothermic neuroprotection based on improved cerebral energy metabolism, using magnetic resonance spectroscopy biomarkers and continuous EEG monitoring. The piglet model of H-I resembles the clinical setting in a neonatal intensive care unit. The observed benefits and safety profile of melatonin support consideration of phase I and II clinical studies of melatoninaugmented therapeutic hypothermia for neonatal encephalopathy. (Robertson NJ, 
Faulkner S, Fleiss B, et al. Melatonin augments hypothermic neuroprotection in a perinatal asphyxia model. Brain 2013 Jan;136(Pt 1):90-105). (Response: Dr Nicola J Robertson. E-mail: n.robertson@ucl.ac.uk).

COMMENT. Melatonin (N-acetyl-5-methoxytryptamine), a naturally occurring hormone secreted by the pineal gland, when administered alone has neuroprotective actions against H-I brain injury in animal models. The present study demonstrates that melatonin augments the neuroprotective effect of hypothermia.

Potential synergistic neuroprotective therapies with hypothermia include inhaled xenon, $\mathrm{N}$-acetylcysteine, erythropoietin, anticonvulsants and cannabinoids, in addition to melatonin. (Cilio MR, Ferriero DM. Semin Fetal Neonatal Med 2010 Oct;15(5):293-8) (Kelen D, Robertson NJ. Early Hum Dev 2010 Jun;86(6):369-77).

\section{SEIZURE DISORDERS}

\section{PROGNOSTIC FACTORS FOR REFRACTORY STATUS EPILEPTICUS}

Researchers at the Mayo Clinic, Rochester, MN studied the outcome and identified prognostic factors for refractory status epilepticus (RSE) in 54 adult patients, median age 52 years [range 18-93]. RSE was defined as generalized convulsive or nonconvulsive status epilepticus that continued despite initial first and second-line therapies. Patients younger than 18 years, anoxic/myoclonic, psychogenic, simple partial, and absence SE were excluded. Of 63 consecutive episodes of RSE, anesthetic agents were used in $55(87.3 \%)$. Duration of drug-induced coma was a mean of 11 days (SD 17.9 days). Cardiac arrhythmias occurred in 21 of 60 episodes $(35 \%)$ and required intervention in 14 of 21 cases (66.67\%). In hospital mortality was $31.75 \%$, in 20 of 63 episodes. Functional outcome at discharge was poor in 48 (76.19\%) episodes. Hospital length of stay was a mean of 27.7 days (SD 37.3 days). Poor functional outcome was associated with drug-induced coma $(\mathrm{p}=0.03)$, cardiac arrhythmias requiring intervention $(\mathrm{p}=0.01)$, and pneumonia $(\mathrm{p}=0.01)$. Prolonged mechanical ventilation was associated with mortality $(\mathrm{p}=0.04)$. Good functional recovery $(\mathrm{p}=0.01)$ followed seizure control without suppression-burst or isoelectric EEG. Functional outcome was not related to age, history of epilepsy, previous SE, type of SE, and anesthetic drug used. (Hocker SF, Britton JW, Mandrekar JN, Wijdicks EFM, Rabinstein AA. Predictors of outcome in refractory status epilepticus. JAMA Neurol 2013 Jan 1;70(1):72-7). (Response: Sara E Hocker MD, Department of Neurology, Mayo Clinic, 200 First St SW, Rochester, MN 55905. E-mail: Hocker.sara@mayo.edu).

COMMENT. In adults with refractory status epilepticus, risk factors for a poor prognosis include the severity of the SE, the need for drug-induced coma, cardiopulmonary complications requiring prolonged mechanical ventilation, and pneumonia. Aggressive EEG suppression does not improve outcome of RSE. Threequarters of adult RSE patients have a poor outcome.

A review of studies of status epilepticus published from 1990-2009 shows that children have a better prognosis than adults, and age and depth of coma are the strongest 